\title{
Philosophiques
}

\section{Les revendications féministes dans le champ religieux québécois : bilan et prospective}

\section{Marie-Andrée Roy}

Volume 21, numéro 2, automne 1994

Les femmes et la société nouvelle

URI : https://id.erudit.org/iderudit/027284ar

DOI : https://doi.org/10.7202/027284ar

Aller au sommaire du numéro

\section{Éditeur(s)}

Société de philosophie du Québec

ISSN

0316-2923 (imprimé)

1492-1391 (numérique)

Découvrir la revue

Citer ce document

Roy, M.-A. (1994). Les revendications féministes dans le champ religieux québécois : bilan et prospective. Philosophiques, 21(2), 433-440.

https://doi.org/10.7202/027284ar
Résumé de l'article

Les femmes engagées dans l'Église constituent les piliers de la vie ecclésiale mais restent des sujettes mineures dans cette institution qui ne leur reconnaît pas, dans les faits, un statut d'égalité avec les hommes. Depuis plus de vingt ans, elles revendiquent des transformations : elles veulent participer à la rédaction des discours officiels, obtenir de meilleurs conditions de travail, mettre de l'avant l'usage du langage inclusif dans les liturgies. Ces femmes, qui détiennent dans une proportion déplus en plus importante une formation en théologie, s'efforcent de conscientiser le clergé à la nécessité de développer avec elles des rapports égalitaires qui impliquent un véritable partage des pouvoirs et des responsabilités. Compte tenu du haut niveau de résistance au changement, il faudrait sans doute que ces femmes acceptent de désobéir à l'ordre clérical et qu'elles osent transgresser les règles de fonctionnement sexiste si elles veulent parvenir à mettre en place une ecclésia égalitaire. 


\title{
Les revendications féministes dans le champ religieux québécois : bilan et prospective
}

\author{
Marie-Andrée Roy
}

\begin{abstract}
RESUME : Les femmes engagées dans l'Eglise constituent les piliers de la vie ecclésiale mais restent des sujettes mineures dans cette institution qui ne leur reconnaît pas, dans les faits, un statut d'égalité avec les hommes. Depuis plus de vingt ans, elles revendiquent des transformations : elles veulent participer à la rédaction des discours officiels, obtenir de meilleurs conditions de travail, mettre de l'avant l'usage du langage inclusif dans les liturgies. Ces femmes, qui détiennent dans une proportion de plus en plus importante une formation en théologie, s'efforcent de conscientiser le clergé à la nécessité de développer avec elles des rapports égalitaires qui impliquent un véritable partage des pouvoirs et des responsabilités. Compte tenu du haut niveau de résistance au changement, il faudrait sans doute que ces femmes acceptent de désobéir à l'ordre clérical et qu'elles osent transgresser les règles de fonctionnement sexiste si elles veulent parvenir à mettre en place une ecclésia égalitaire.
\end{abstract}

ABSTRACT : Although women constitute the pillars in the ongoing life of the Church, their status is not equal to that of men and they play a secondary role in the institution. For at least the last twenty years, women have been demanding changes such as : the participation in the elaboration of official statements, the betterment of working conditions and the use of inclusive language in liturgical celebrations. Many women, who have a theological formation, endeavour to make the clergy realize the necessity of developing relationhips of real equality. This implies a concrete division of power and responsability. Given the degree of resistance in the institutional Church, women may have to accept disobedience to clerical power, thus breaking the rules of a sexist functioning in order to establish an Ecclesia of equality.

Pourquoi traiter des revendications féministes dans le champ religieux ? Est-ce encore pertinent de s'intéresser à un champ qui a connu depuis trente ans une forte désaffection de sa clientèle? A mon avis, l'étude de la question des femmes dans le champ religieux reste épistémologiquement importante 
pour comprendre la situation des femmes dans notre culture. Escamoter le religieux en affirmant qu'il n'a plus de signification sociale c'est emprunter un raccourci qui produit une distorsion dans l'analyse de la situation des femmes dans la société québécoise actuelle. Je m'intéresse au champ religieux parce qu'il est un des lieux où se construisent les rapports hommes/femmes dans notre société, qui met de l'avant une lecture de ce que signifie être femme et homme aujourd'hui, qui génère, par sa symbolique organisationnelle et discursive, une représentation de la féminité qui imprègne l'imaginaire social.

\section{Le statut des femmes dans l'Église}

Entrons dans le vif du sujet. Peut-on discerner des revendications féministes dans le champ religieux catholique québécois? Divers travaux tendent à le confirmer ${ }^{\mathrm{I}}$. Mais, à entendre le discours des personnes impliquées à l'intérieur de l'organisation ecclésiale, il n'y aurait pas de revendications féministes. Il y aurait plutôt des souhaits, des recommandations, des attentes, des espérances, des souffrances, etc. qui sont énoncés par les femmes et que l'Église écouterait avec attention et bienveillance. Cette série de substantifs pour contourner presque systématiquement le mot « revendications » indique bien la présence d'un interdit autour de ce mot. Interdit parce qu'il n'est pas question de reconnaitre l'existence de rapports de pouvoir, de rapports de force entre les personnes de cette institution. Il n'y aurait que des baptisés qui exercent des fonctions différentes, chacun selon sa vocation. Les clercs, détenteurs de l'autorité, n'auraient pas de pouvoir; en fidélité à l'appel reçu, ils rempliraient un service dans un esprit d'obéissance au successeur de Pierre.

Cette occultation des rapports de pouvoir a un impact considérable sur la compréhension qu'ont les femmes de leur situation à l'intérieur de l'organisation, sur le type de relations qui prévalent entre les hommes-clercs et les femmes-laïques et enfin sur les stratégies que ces dernières mettent en œuvre pour signifier leur aspiration à des rapports égalitaires. Le puissant interdit qui pèse autour de la reconnaissance des rapports de pouvoir, rend illégitime tout discours ou pratique de revendications des femmes dans l'Église. C'est pourquoi, très souvent, elles se contentent de formuler des souhaits.

Mais je ne soutiens pas par là que les femmes se soumettent toutes inconditionnellement au pouvoir clérical et qu'elles se complaisent dans la subordination. Je pense qu'il existe, chez une fraction importante des femmes directement engagées dans l'Église, une conscience de l'inégalité qu'elles vivent et une volonté de parvenir à des rapports différents. Mais la situation

I. Voir notamment les textes de Lise Baroni, « L'émergence créatrice des femmes engagées dans l'Église ». Femmes. Pour quel monde? Dans quelle Eglise?, Ottawa, Conférence Religieuse Canadienne, 1985, p. 43-65, et Marie-Andrée Roy, « Les revendications des femmes dans l'Eglise », dans Souffles de fermmes. Lectures féministes de la religion, Montréal, Editions Paulines, 1989, p. $29-72$. 
des femmes dans l'Eglise est à la fois complexe et paradoxale. En effet, elles sont actuellement des milliers à travailler comme salariées et comme bénévoles dans cette organisation. Les enquêtes que j'ai effectuées pour ma thèse de doctorat et à l'intérieur de l'équipe de recherche d'Anita Caron ${ }^{2}$ me permettent d'affirmer qu'elles jouent un rôle absolument indispensable dans l'Eglise. Elles ne se limitent pas à être des consommatrices des services religieux, elles constituent les piliers des organisations paroissiales et diocésaines. On les retrouve partout, en majorité d'ailleurs, dans des fonctions pastorales, liturgiques, administratives, caritatives et autres. Le travail qu'elles accomplissent est loin d'être inintéressant. Il fait appel à leurs qualités d'organisatrices, d'animatrices, d'éducatrices et d'administratrices, etc. Elles forment souvent des équipes de travail dynamiques, de plus en plus scolarisées. Ces engagements représentent, pour plusieurs d'entre elles, une manière de rompre avec l'isolement de la maison familiale, une possibilité de prendre la parole, d'acquérir plus de confiance en soi et de travailler en équipe.

Mais ces piliers de la vie ecclésiale restent des sujettes mineures dans une Église qui ne leur reconnaît pas, dans les faits, un statut d'égalité avec les hommes. Juridiquement, les femmes sont subordonnées aux clercs. Dans le cadre de leurs engagements, elles vivent, dans bien des cas, une véritable situation de domination et d'exploitation parce que le travail qu'elles accomplissent n'est pas reconnu à sa juste valeur et qu'elles sont fréquemment désappropriées des réalisations de ce travail. Exclues de toutes les fonctions sacrées, elles incarnent, individuellement et collectivement, le sexe féminin tentateur, le sexe pécheur dont il faut se distancier pour accéder au sacerdoce. Elles sont infantilisées au plan de l'agir moral. En effet, les autorités cléricales n'ont pas abandonné leur prétention à régir la conscience des femmes et à contrôler leur corps. Bref, l'aliénation des femmes dans l'Eglise est un fait irréfutable même s'il coexiste avec une pratique d'implication et d'engagement qui est loin d'être dénuée de sens.

\section{Les revendications des femmes dans l'Église}

Mais qu'elles sont ces revendications des femmes, car il s'agit bien de revendications même si elles portent des appellations qui tendent à occulter leur véritable nature?

La revendication centrale qui traverse, depuis plus de vingt ans, l'ensemble des autres préoccupations des femmes dans l'Église catholique québécoise, c'est la reconnaissance de leur égalité avec les hommes, de leur statut de personne à part entière. Cette égalité, elles la veulent non seulement devant

2. Ma thèse a pour titre Les femmes et le pouvoir dans l'Eglise. Le cas de l'Eglise catholique au Québec, de 1970 à nos jours, Université du Québec à Montréal, 1992. L'équipe de recherche a publié, sous la direction d'Anita Caron. Femmes et pouvoir dans l'Eglise, Montréal, V.L.B. éditeur, 1991, 254 P. 
Dieu, comme l'institution la leur reconnaît, mais également devant les hommes, c'est-à-dire dans les faits et gestes de l'organisation ecclésiale. Cette revendication insistante indique bien que des femmes sont conscientes de leur statut d'infériorité, qu'elles ne le considèrent pas comme légitime et qu'elles entendent bien le faire changer. En cela, elles font leur le grand principe qui anime le féminisme contemporain depuis plusieurs décennies.

La revendication pour l'égalité s'accompagne d'une série d'autres doléances qui suggèrent clairement que leurs formulatrices perçoivent la nécessité de traduire cette dite égalité dans la réalité concrète de l'organisation ecclésiale, que l'égalité implique l'accès à des fonctions comportant des pouvoirs et qu'il faut des moyens pour l'atteindre.

\subsection{Pour une égalité effective}

Afin de rendre tangible l'égalité, nombre de femmes revendiquent leur participation à la rédaction des discours officiels de l'Église, notamment ceux qui ont trait aux questions morales. Elles désavouent ainsi implicitement le discours de l'Église sur ce sujet. Elles se perçoivent comme des personnes moralement responsables capables de développer et de proposer une réflexion éthique signifiante.

De plus en plus de travailleuses dans l'Église entendent faire reconnaître leur égalité par l'intermédiaire des conditions de travail qui les régissent. L'augmentation continue du nombre de femmes à l'emploi de l'institution a pour effet de favoriser une plus grande formalisation des contrats de travail et le développement de réseaux de solidarité entre ces travailleuses. Mais, à notre avis, le respect des contrats de travail demeure encore très aléatoire et, dans bien des cas, soumis à l'arbitraire des employeurs locaux. Le principe même de l'égalité entre toutes les catégories d'intervenants (hommes-clercs et femmeslaïques) demeurera inaccessible tant et aussi longtemps que l'Église sera définie en termes hiérarchiques et masculins. Les réseaux de solidarité qui s'amorcent depuis quelque temps apparaissent assez fragiles et dépourvus de véritables moyens d'action pour exercer une influence significative.

Pour bien des femmes engagées en Église, l'égalité devrait également se traduire par l'usage du langage inclusif, notamment dans les liturgies. Elles sont parvenues à quelques avancées timides sur ce sujet. Aux « biens chers frères » des débuts des homélies s'ajoute maintenant la reconnaissance des «biens chères sœurs ». Mais la logique du langage inclusif devrait soulever des questions qui dépassent de beaucoup les quelques adaptations formelles consenties jusqu'ici. Elles devraient interroger le caractère exclusivement masculin de toute la hiérarchie. Pourquoi les expressions comme «prêtresse », « femme évêque », « cardinale » et « papesse » semblent-elles appartenir à la science-fiction ou à l'univers religieux paien ? L'usage du langage inclusif devrait susciter une confrontation avec le discours théologique sur le « Dieu dit de nos Pères », ce Dieu dont on a fait la quintessence du masculin. Bref, c'est 
tout l'édifice hiérarchique et symbolique qui pourrait faire l'objet d'une remise en cause radicale. Mais il ne semble pas que l'on en soit là.

\subsection{Des moyens pour y parvenir}

Les moyens les plus fréquemment proposés pour faire avancer l'égalité sont, pour les femmes elles-mêmes, l'accès à une formation théologique et le travail de conscientisation du clergé.

Les femmes engagées en Église souhaitent fortement accéder à une formation théologique qui tienne compte de leurs besoins, de leurs aspirations, de leurs expériences. Elles ne veulent pas d'un instrument de cléricalisation des laïcs. Cette crainte n'est pas farfelue parce qu'il ne faut pas remonter très loin dans l'histoire pour découvrir que la théologie était un savoir réservé aux clercs. C'est seulement depuis quelque trente ans que les laïcs peuvent étudier la théologie à l'université. La présence de professeurs laïcs est donc encore plus récente et celle de femmes professeures demeure marginale même si, depuis quelques années, la clientèle étudiante est composée d'une nette majorité de femmes (plus de 6o\% dans certaines institutions).

L'Église a de plus en plus tendance à exiger que les personnes qu'elle embauche détiennent une formation théologique; en même temps, des diocèses, en concertation avec des centres universitaires, ont commencé à se doter de programmes de formation théologique qui s'adressent aux personnes impliquées dans leur diocèse. Dans certains cas l'employeur devient le formateur. Ce cumul des rôles peut soulever des interrogations sur le type de formation proposée. Enfin, les pressions de Rome ont tendance à s'accentuer ces dernières années pour préserver l'orthodoxie du discours théologique. Dans une telle conjoncture, le risque que la théologie devienne un instrument de cléricalisation des femmes semble fondé.

Quant au travail de conscientisation du clergé, il retient l'attention des intervenantes depuis très longtemps. Plusieurs, par exemple, réclament que des femmes soient associées à la formation des futurs prêtres. Elles pointent clairement la source des difficultés sans pousser la logique jusqu'au bout. Le célibat obligatoire et l'exclusion des femmes du sacerdoce font des prêtres une caste qui se définit en l'absence des femmes quand ce n'est pas à leur encontre. Cette même caste est par ailleurs appelée à travailler quotidiennement avec les femmes tout en s'en protégeant. Cet illogisme au plan des relations humaines peut engendrer bien des aberrations et constitue, à mon sens, une situation propice au développement de la misogynie. On comprendra que les quelques efforts consentis à la conscientisation du clergé ne peuvent pas extirper un problème qui a pour origine la contrainte au célibat et l'exclusion d'un sexe pour l'exercice des fonctions sacerdotales.

\section{3 le partage des pouvoirs}

L'égalité signifie aussi, pour un certain nombre de femmes, la possibilité de partager des responsabilités, d'exercer des pouvoirs. 
C'est autour de ce noyau central du pouvoir que se corse le débat. Le principe de l'égalité voudrait que les femmes puissent exercer, à compétence égale, des responsabilités à tous les paliers de l'organisation ecclésiale et qu'il n'y ait pas de discrimination fondée sur le sexe. Ce principe implique aussi l'accès à tous les ministères. Si un petit nombre de femmes défend cette idée, plusieurs hésitent à la mettre de l'avant. En fait le fameux débat égalitél différence a rejoint les femmes dans l'Église. Trois grandes tendances se dessinent alors.

Pour certaines, l'égalité constitue avant tout une question de principe qu'il faut faire reconnaître et appliquer dans toute forme d'organisation y compris dans l'Église catholique et ses ministères ordonnés. Ces femmes s'inspirent directement des arguments qui ont conduit les féministes égalitaires à revendiquer la place qui leur revient dans les partis politiques, les entreprises, etc. D'autres disent vouloir exercer un sacerdoce à la manière des femmes ou avoir des ministères qui leur sont propres. Ces ministères féminins qui, la plupart du temps, sont définis comme « non ordonnés » pourraient, je pense, recevoir la bénédiction de Rome. Cette instance se montre toujours désireuse de reconnaître et de valoriser la vocation spécifique de la femme pourvue qu'elle n'empiète pas sur le territoire sacerdotal. Certaines femmes soutiennent enfin que la promotion de la stricte égalité entraîne la reproduction de l'Église cléricale et masculine que nous connaissons; elles remettent en question le modèle masculin de l'organisation ecclésiale et la manière d'y vivre le sacerdoce. Elles ne veulent pas exercer de ministère sacerdotal tel que conçu actuellement par et dans l'Église telle qu'elle est aujourd'hui. Par ailleurs la formulation des alternatives demeure assez vague.

L'absence d'une vision partagée quant au statut et au rôle des femmes ne favorise pas l'avènement d'actions concertées en Eglise, voire la mise en place de réseaux de solidarité efficaces. La fragile quête de l'égalité semble encore plus compromise quand on examine les taux de satisfaction des travailleuses à l'emploi de l'Église. Même si les femmes sont exclues de nombreux postes de responsabilités, c'est dans une proportion très élevée (plus de 9o\%) qu'elles se déclarent assez et très satisfaites des tâches qu'elles exercent dans l'Église et du niveau de responsabilités qu'elles occupent ${ }^{3}$.

\section{Prospective}

Sur ce terrain miné de contradictions, donnons quelques éléments de prospective.

Les revendications des femmes dans l'Église ont mis du temps à faire surface et suivent de quelques années celles des femmes dans la société civile.

3. Voir à ce sujet le rapport de recherche publié par Anita Caron et Marie-Andrée Roy, Fernmes, formation théologique et emplois, Centre de recherches féministes, UQAM, 1992, $24 \mathrm{p}$. 
Elles se modèlent assez souvent sur les revendications mises de l'avant par le mouvement social de libération des femmes. Cette situation nous indique comment les idées et les valeurs qui circulent dans le monde séculier parviennent à imprégner le monde religieux. C'est d'ailleurs en période d'intense activité du mouvement des femmes au Québec que les revendications des femmes dans l'Église ont reçu le meilleur accueil de la part des autorités ecclésiales. Dans la conjoncture actuelle les avancées apparaissent de plus en plus hypothétiques. Voici pourquoi.

Le concept utilisé de façon prépondérante dans l'Église pour évoquer les relations hommes/femmes est celui du « partenariat ». Il est mis de l'avant tant par des femmes engagées dans cette institution que par des clercs. Ce concept de partenariat est emprunté au monde des affaires et de la politique, qui s'en sert pour développer un nouveau type de relations de travail, favoriser l'implication de toutes les parties en présence et dynamiser leur organisation. Il s'agit d'un concept novateur, apparenté au libéralisme économique, mais singulièrement porteur de « vices cachés ». Je m'explique.

Le partenariat peut être juste et équitable quand il implique des partenaires qui ont les mêmes droits. Une association de partenaires inégaux ne constitue pas un partenariat mais un rapport de subordination, voire même d'exploitation. L'usage du terme « partenariat » peut suggérer l'idée d'égalité tout en consacrant dans les faits l'inégalité. C'est ce qui se passe dans l'Église. Il est difficile de revendiquer l'accès à un statut d'égalité, avec tout ce que ce concept implique, quand les femmes-laïques et les hommes-clercs sont censés avoir déjà mis en place un partenariat qui suppose l'harmonisation de leurs rapports.

Ce n'est pas tout. La force de l'Église catholique est aussi liée à sa capacité d'obtenir l'obéissance de ses membres à tous les échelons de son organisation. Les religieux font des vœux d'obéissance, les vicaires doivent « obéissance »à leur curé, le curé à l'évêque et l'évêque, lors de son intronisation, promet d'obéir au pape. Les femmes quant à elles, ont appris à obéir dès leur prime jeunesse. Cette règle de l'obéissance est profondément intériorisée. Elle semble avoir pour effet de déresponsabiliser les différents paliers de la hiérarchie cléricale. En effet, à chaque palier on refuse de prendre la responsabilité du problème et on soutient que la solution relève de quelqu'un d'autre. Le prêtre invoque son curé, le curé affirme que ce n'est pas de son ressort, l'évêque s'en remet au pape qui, lui, déclare que, devant Dieu, il ne peut pas accepter l'ordination des femmes.

Pour bon nombre des femmes impliquées dans l'Église, l'obéissance semble être un élément constitutif de leur féminité. Pas même nécessaire de faire de vœu, être femme cela veut dire obéir. Rappelons-nous ce qui est arrivé à la première qui a désobéi. Sur cette toile de fond, l'introduction de changements semble fortement compromise. De tels changements impliqueraient, de la part de ces femmes, une joyeuse désobéissance à l'ordre clérical, la perception de l'illégitimité du pouvoir exclusivement masculin, la transgression des 
règles de fonctionnement sexistes. Or, tout est mis en place pour assurer l'immuabilité de l'ordre clérical dominant et la perpétuation d'une féminité faite d'abnégation héroïque et d'obéissance «volontaire » au Dieu toutpuissant.

Les Américaines ont bien montré comment le mouvement féministe fait face, depuis quelque temps, à un véritable «backlash ». Il semble qu'il en soit de même dans l'Église québécoise. Même si le mouvement des femmes dans l'Église est plus récent et d'ampleur nettement plus limitée, il apparaît déjà aux prises avec un certain «backlash ». Les structures mises en place pour assurer l'avancement de la situation des femmes, comme, par exemple, le Réseau des répondantes à la condition féminine, semblent connaître un certain essoufflement. Monte de plus en plus la rumeur cléricale qui dit sa lassitude devant les revendications des femmes. Les mouvements de résistance du clergé devant la nomination de telle ou telle femme à un poste de responsabilité se font de plus en plus fréquents, etc. Bref, après le temps de la tolérance, des mea culpa, vient celui de la relancée de l'autorité cléricale. Cette relancée coïncide avec la nomination d'évêques plus sensibles aux perspectives romaines.

A mon avis, on s'oriente donc vers un renforcement du pouvoir clérical masculin et vers une accentuation d'une attitude de domination à l'égard des femmes dans l'Église. Il existe bien ici et là quelques pratiques alternatives, des regroupements porteurs d'aspirations au changement mais, dans l'ensemble, on n'est pas parvenu à ébranler les fondements symboliques du pouvoir clérical ni à désamorçer le discours définisseur des femmes et de la féminité qui continue de prévaloir et de s'infiltrer dans les interstices des imaginaires. En même temps, les femmes sont de plus en plus nombreuses à travailler directement sous l'autorité de clercs comme bénévoles ou salariées. On n'a jamais eu une telle proportion de femmes laïques à l'emploi de l'Église et le phénomène est appelé à se maintenir pour un certain temps. Les conditions sont réunies pour que se reproduise la domination des femmes.

On pourrait toujours dire qu'une telle situation n'est pas grave et qu'elle ne concerne que les femmes engagees dans cette institution. On ne peut soutenir un tel point de vue que si on fait fi de l'influence du religieux dans notre société et de l'existence de rapports Église/État non négligeables.

Les idéaux d'égalité n'apparaissent pas voués à un avenir très prometteur dans l'Église. Et pourtant, il me semble que la discrimination sexiste qui fait structurellement partie de l'organisation ecclésiale devrait faire l'objet de la plus vive réprobation sociale. Si l'Eglise s'avisait d'être raciste dans le choix de ses futurs prêtres, elle serait vigoureusement dénoncée et avec raison. Mais elle pratique un véritable racisme fondé sur le sexe et on considère que cette situation ne regarde pas la société, qu'elle ne concerne que les membres de cette Église. 